\title{
VAC.I3 - Evaluation of different adjuvant formulations for development of an inactivated Yellow Fever vaccine candidate
}

Ana Carolina Cajaraville ${ }^{1}$; Mariana Pierre ${ }^{1}$; Renata Carvalho Pereira ${ }^{1}$; Patricia da Costa Neves ${ }^{1}$; Elena Caride ${ }^{1}$; Sheila Maria Barbosa de Lima ${ }^{1}$; Luciane Pinto Gaspar ${ }^{1}$; Marco Alberto Medeiros ${ }^{1 *}$. 1Fiocruz/Bio-Manguinhos.

Introduction: Yellow Fever vaccine is one of the most successful vaccines ever developed. Nonetheless, restrictions of administration to immunosuppressed and rare adverse events are drawbacks that stimulate the development of safer non-live approaches. As safety increases, however, immunogenicity decreases due to the lack of viral replication. In this context, adjuvants are key elements to activate innate immunity and shape the desired adaptative responses and protection. Adjuvants of different mechanisms of action have been studied and we selected candidates of each class: antigen carriers $\left(\mathrm{Al}(\mathrm{OH})_{3}\right.$ and Addavax) and immune potentiators (Flagellin). In this study we intend to identify promising adjuvants for development of an inactivated yellow fever virus (IYFV) vaccine candidate, in preclinical evaluation in mice model.

Objective: The aim to use IYFV as a model to identify promising adjuvant formulations.

Methodology: C57BL/6 mice were immunized with IYFV formulated with different adjuvants: $\mathrm{Al}(\mathrm{OH})_{3} 0,3 \%$; Addavax ${ }^{\otimes}$ from InvivoGen (MF59-like squalene) or a combination of $\mathrm{Al}(\mathrm{OH})_{3}$ 0,3\%, and Flagellin (TLR5 agonist) in 2-dose (Do and D28) or 3-dose (D0, D14 and D28) schedule. After immunization, mice were challenged by lethal intracerebral (IC) inoculation of YFV to determine survival rates and sera were analysed by ELISA and PRNT for detection of total IgG and neutralizing antibodies against YFV. In addition, IgG subtypes were determined in order to address antibodies' functions.

Results: IYFV formulated with Addavax was able to induce $100 \%$ of protection after 2-dose regimen against $25 \%$ survival induced by alum formulation. The best survival rate achieved with $\mathrm{Al}(\mathrm{OH})_{3}$ was $78 \%$ in 3-dose regimen. The combination $\mathrm{Al}(\mathrm{OH})_{3}+$ Flagellin didn't show increment in survival rates. Both formulations (with Addavax and $\mathrm{Al}(\mathrm{OH})_{3}$ ) were able to induce high titers of IgG against YF; however, neutralizing antibodies levels were borderline on prechallenge time. The analysis of IgG subtypes revealed a predominance of IgG2a (associated with better neutralizing capacity) in the animals immunized with the attenuated vaccine, against a predominance of IgG1 in the groups immunized with the experimental formulations. The equivalent analysis in the survivors of each group, however, showed an increase of neutralizing antibodies and IgG2a titers after the boost effect of the challenge with the YFV.

Conclusion: The use of the Addavax ${ }^{\circledast}$ as adjuvant for yellow fever non-live candidates comes up as a promising alternative to achieve protection and dose-spare. However, the immune responses induced by this experimental formulation differ from the correlates of protection originally described for the attenuated vaccine (high neutralizing antibody titers). As the survival rates and increase in neutralizing antibody titers after IC challenge clearly demonstrate an anamnestic response of the animals immunized with both addavax and alum-based formulations, further investigation of the mechanisms of action of these experimental formulations is ongoing to better characterize other possible features of immune responses involved with protection in this model.

Keywords: inactivated Yellow Fever vaccine candidate; adjuvant formulations; yellow fever non-live candidates 